CWRU-P17-96

November 1996

\title{
QUANTUM HAIR, INSTANTONS, AND BLACK HOLE THERMODYNAMICS: SOME NEW RESULTS
}

\author{
Lawrence M. Krauss \ and Hong Liu \\ Department of Physics \\ Case Western Reserve University \\ 10900 Euclid Ave., Cleveland, OH 44106-7079
}

\begin{abstract}
We present results obtained by a consideration of the non-classical energy momentum tensor associated with Euclidean Instantons outside the event horizon of black holes. We demonstrate here how this allows an analytic estimate to be made of the effect of discrete quantum hair on the temperature of the black hole, in which the role of violations of the weak energy condition associated with instantons is made explicit, and in which the previous results of Coleman, Preskill, and Wilczek are extended. Last, we demonstrate how the existence of a non-classical electric field outside the event horizon of black holes, uncovered by these authors, can be identified with a well-known effect in the Abelian-Higgs model in two dimensions. In this case, there is a one-to-one connection between the discrete charge of a black hole and a topological phase in two dimensions.
\end{abstract}

\footnotetext{
${ }^{1}$ also Department of Astronomy
} 


\section{Introduction}

Our understanding of how gravity and quantum mechanics may be reconciled has changed considerably as a result of semiclassical considerations. Arguably the most famous example involves Hawking radiation, with its associated implications for black hole thermodynamics. Indeed, the very process of black hole evaporation, which is made possible in principle by the effects of quantum fluctuations near the event horizon of a black hole, has challenged the foundations of quantum mechanics itself. If Hawking radiation by a black hole is purely thermal, with no hidden correlations between outgoing emitted particles, then complete evaporation of a black hole would allow pure quantum mechanical states to evolve into mixed states, violating unitarity, and leading to the loss of information (i.e. [1], 2])

Much work has been carried out in the past decade aimed at addressing this potential problem. No definitive solution is yet at hand, but a lot has been learned during the process about such things as state counting and black hole entropy, possible new Planck Scale phenomena, and phenomena in higher dimensions.

At the same time, several interesting results have been obtained which involve essentially only phenomena far below the Planck Scale. In particular, it has recently been established that black holes can harbor "quantum hair" (i.e. 3, 4, 51) - that is, quantum mechanical observables can be associated with black holes beyond those allowed by the classical "no hair theorems" [07, 8, 9] (for recent discussions, see [10]).

We first briefly describe a canonical example of gauged quantum hair [⿴囗⿴囗丨 . Consider an abelian U(1) gauge theory containing two matter fields $\eta$ and $\phi$ 
with charge $N e$ and $e$ respectively. If the field $\eta$ condenses at some energy scale $v$, then the gauge field will become massive by the Higgs mechanism, and below this scale the effective theory involving only the light field $\phi$ will have a residual discrete $Z_{N}$ symmetry.

At the same time, this low energy broken symmetry theory also can contain stable strings threaded by magnetic flux $2 \pi / N e$. The scattering of $\phi$ quanta, with charge $e$ from such strings is dominated the Aharonov-Bohm effect [11, 12]. Since such scattering involves quantum phases uniquely determined by the product of charge and flux, a determination of the total charge modulo $\mathrm{N}$ which scatters off the string is thus possible. Since the quantum phases in question are global quantities, a $\phi$ quanta which falls into a black hole will be measurable as such even after it falls inside the event horizon of the black hole [4, 13, 14, 15]. Such a charged black hole therefore has "quantum hair".

The proof of the existence of non-classical gauge hair on black holes 四, [13, 5] which could obviate the famous no-hair theorems for black holes led to hopes that such non-classical hair might alleviate the black hole information loss problem. However, while such hopes evaporated about as quickly as a small black hole might, efforts soon focussed on exploring whether quantum hair might have any other observable effects.

Indeed, given the semiclassical relationship between entropy, area, and temperature for black holes, it is natural to expect that any restriction on the number of microstates associated with a given classical black hole macrostate, such as would occur if one could measure additional black hole quantum numbers, would have a related effect on the black hole's entropy 
and temperature. This possibility was explored most recently in a beautiful series of papers by Coleman, Preskill and Wilczek [5, 6]. These authors uncovered an (exponentially small) effect on temperature, but surprisingly the sign of the temperature change depended upon the relative scale of the spontaneous symmetry breaking associated with the quantum hair compared to the inverse size of the black hole event horizon. More interestingly perhaps, they also uncovered a new observable associated with quantum hair: a non-classical electric field could exist and be measured outside of the event horizon.

Since these calculations were performed in the context of the Euclidean Action in curved space, it would be of some interest to examine these effects in a formalism more closely related to Minkowski space methods, where presumably some additional physical insights might arise. Recently, we outlined such an approach [16]. Here we extend the arguments outlined in our earlier letter, and also provide a more detailed derivation of both our original and several new results. In section 1 we outline our formalism. In section 2 we review the application of this method to the case of a Reissner-Nordstrom black hole. In the next section we discuss the Abelian Higgs theory in the thin string limit, and then follow this with a detailed discussion of the thick string limit, where several additional subtleties arise. Finally we describe how the results obtained by CPW can be recast in the light of similar results from two dimensional quantum field theory. In this case, there is a one to one mapping between discrete charge on a black hole, and a topological phase. We conclude with a brief discussion of possible implications of our results. 


\section{Formalism}

Following CPW's demonstration that a non-classical field exists outside the event horizon of a black hole endowed with discrete gauge hair, it then seems reasonable to focus on the energy momentum tensor outside the black hole. A Minkowski-space formalism which does just this was developed by Visser [17] for treating " dirty" black holes, where non-zero matter fields exist outside the event horizon. Unfortunately, since the electric field associated with discrete hair is non-classical - i.e.it is not a solution of the coupled vacuum EinsteinMaxwell equations - standard methods such as Visser's, which require such solutions, cannot be applied directly.

It is true, however, that while the electric field generated outside the event horizon is not a solution of the Minkowski field equations, the individual instantons whose contributions sum to produce such a field are solutions of the coupled Euclidean Einstein-Maxwell equations. As a result, we have utilized [16] a hybrid approach, in which we reproduce the Visser formalism in Euclidean space, and then focus on the effect of individual instantons. We derive here several key results which we will then employ to analyze a variety of black hole configurations with and without discrete hair.

The Euclidean spacetime metric generated by a static spherically symmetric distribution of matter can be put in the form:

$$
d s^{2}=e^{-2 \phi(r)}\left(1-\frac{b(r)}{r}\right) d t^{2}+\left(1-\frac{b(r)}{r}\right)^{-1} d r^{2}+r^{2} d \Omega^{2}
$$

With the assumption that the metric has an asyptotically flat geometry and an event horizon, boundary conditions can be imposed as:

$$
\phi(\infty)=0, \quad b(\infty)=2 G M_{B H}, \quad b\left(r_{H}\right)=r_{H}
$$


where $M_{B H}$ is the mass of the black hole and $r_{H}$ is the horizon size. Einstein's equations,

$$
R_{\mu \nu}-\frac{1}{2} g_{\mu \nu} R=8 \pi G T_{\mu \nu}
$$

with

$$
T_{\mu \nu}=\frac{2}{\sqrt{g}} \frac{\partial\left(\mathcal{L}_{E} \sqrt{g}\right)}{\partial g^{\mu \nu}}
$$

can then be solved formally to give $b(r)$ and $\phi(r)$ in terms of the components of the energy momentum tensor:

$$
\begin{aligned}
& b(r)=r_{H}-8 \pi G \int_{r_{H}}^{r} d r^{\prime} \rho\left(r^{\prime}\right) r^{\prime 2} \\
& \phi(r)=4 \pi G \int_{r}^{\infty} d r^{\prime} \frac{(\tau-\rho) r^{\prime}}{1-b\left(r^{\prime}\right) / r^{\prime}}
\end{aligned}
$$

In the above we define

$$
T_{t}^{t}=\rho, \quad T_{r}^{r}=\tau, \quad T_{\theta}^{\theta}=T_{\varphi}^{\varphi}=-\mu .
$$

which satisfy the conservation law

$$
\tau^{\prime}+(\tau-\rho)\left(-\phi^{\prime}+\frac{1}{2}\left(\ln \left(1-\frac{b(r)}{r}\right)\right)^{\prime}\right)+\frac{2}{r}(\mu+\tau)=0
$$

Using equations (11) (2), the Hawking temperature and the horizon size of the black hole can now be expressed as (i.e. see [17]):

$$
\begin{aligned}
\frac{1}{\beta \hbar} & =\frac{1}{4 \pi r_{H}} e^{-\phi\left(r_{H}\right)}\left(1-b^{\prime}\left(r_{H}\right)\right) \\
& =\frac{1}{4 \pi r_{H}} \exp \left(-4 \pi G \int_{r_{H}}^{\infty} d r \frac{(\tau-\rho) r}{1-b(r) / r}\right)\left(1+8 \pi G \rho_{H} r_{H}^{2}\right) \\
r_{H} & =2 G M_{B H}+8 \pi G \int_{r_{H}}^{\infty} d r \rho r^{2}
\end{aligned}
$$

When the external matter contribution to the geometry is much smaller than that of the black hole, equations (5) and (6) can be systematically 
expanded and the lowest order corrections to black hole thermodynamics can then be obtained. Now define

$$
\begin{aligned}
A & =\frac{8 \pi G}{2} \int_{r_{H}}^{\infty} \frac{\rho-\tau}{r-r_{H}} r^{2} d r \\
B & =8 \pi G \rho_{H} r_{H}^{2} \\
m & =4 \pi \int_{r_{H}}^{\infty}(2 \mu+\rho-\tau) r^{2} d r
\end{aligned}
$$

Expanding (5) and (6) to first order in $A, B$, and $\left(m / M_{B H}\right)$ and using the conservation law (3) we reach:

$$
\begin{aligned}
\frac{1}{\beta \hbar} & =\frac{1}{4 \pi} \frac{2 G M_{B H}}{r_{H}^{2}}\left(1+\frac{m}{M_{B H}}\right)+\text { higher order corrections } \\
r_{H} & =2 G M_{B H} \frac{1+\frac{m}{M_{B H}}}{1+A+B}+\text { higher order corrections }
\end{aligned}
$$

(Note that (8) is equivalent to the Bardeen-Carter-Hawking mass theorem [18].)

Plugging equation (8) into (8), we get an expression for $\beta \hbar$ in terms of only the components of the energy momentum tensor and $M_{B H}$ [16]: ( In this order, $r_{H}$ can be replaced by its lowest order piece, i.e. $r_{H}=2 G M_{B H}$.)

$$
\beta \hbar=8 \pi G M_{B H}\left(1+\frac{m}{M_{B H}}-2(A+B)+\text { higher order corrections }\right) .
$$

For further reference, it is useful to write out this expression in more detail. In particular,

$$
\frac{m}{M_{B H}}-2(A+B)=-\frac{8 \pi G}{r_{H}} \int_{r_{H}}^{\infty} d r\left[\left(4 \mu r r_{H}-2 \mu r^{2}\right)-(\rho-\tau) r\left(r-r_{H}\right)\right]
$$

It is clear that the sign of the correction to the black hole temperature, for fixed mass, depends upon the relative sign of the term $m / M_{B H}-2(A+B)$. Using this fact, we can further explore the result of CPW that discrete charge 
on a black hole can either raise or lower the black hole temperature, depending upon the ratio of the gauge symmetry breaking scale to the inverse horizon size of the black hole, a somewhat surprising result at first sight. Indeed, this is perhaps even more surprising when one considers that all forms of classical matter which satisfy the Weak Energy Condition (WEC) also satisfy the relation $m / M_{B H}-2(A+B) \geq 0$. This is one example of a general result that classical matter outside a black hole can only lower its temperature[17]. By framing the discussion in terms of the quantity $m / M_{B H}-2(A+B)$, our analysis will allow us to establish an explicit connection between the WEC and the effects of instantons, about which we will have more to say later.

In the following sections we use the above results to examine the effect of various instanton contributions to the temperature of a black hole in the semiclassical limit. Following Coleman, Preskill, and Wilczek (CPW), we explore first the case of an unbroken $U(1)$ theory with unshielded electric charge, and then the broken theory involving discrete hair. A great deal of physical insight can be gained by focussing on the sometimes subtle distinctions between these cases.

\section{The Euclidean Reissner-Nordstrom Black Hole}

A Euclidean Reissner-Nordstrom black hole provides a simple application of the above formalism, and one to which we shall be able to compare our later results. ( In what follows, we will assume $M_{p l} / M_{B H} \ll 1$.) We recall that this is a Euclidean solution of the field equations which is periodic in imaginary time with period $\beta \hbar$. Moreover, because it describes a configuration with 
finite charge, the temporal component of the gauge field is restricted by a gauge constraint (i.e. see [5]):

$$
\left.e \int_{0}^{\beta \hbar} d t A_{t}\right|_{r=\infty}=\omega
$$

where $\omega$ is a constant related to the value of the electric charge, as we will explicitly derive momentarily.

The action of this system is:

$$
S_{E}=\int d^{4} x \sqrt{g}\left(\mathcal{L}_{e m}-\frac{1}{16 \pi G} R\right)+\frac{1}{2}(\beta \hbar) M_{B H}
$$

with

$$
\mathcal{L}_{e m}=\frac{1}{16 \pi} g^{\mu \lambda} g^{\nu \sigma} F_{\mu \nu} F_{\lambda \sigma}
$$

The stationary point solution is:

$$
\begin{array}{rc}
A_{t} & =\frac{\omega}{\beta \hbar e}\left(1-\frac{r_{H}}{r}\right) \\
F_{r t} & =\quad \frac{\omega}{\beta \hbar e} \frac{r_{H}}{r^{2}} \\
\phi(r) & =0
\end{array}
$$

The energy momentum tensor is given by:

$$
\rho=\tau=\mu=\frac{1}{8 \pi} F_{r t}^{2} \propto \frac{1}{r^{4}}
$$

Now, interestingly, from (10),

$$
2(A+B)-\frac{m}{M_{B H}}=\frac{8 \pi G}{r_{H}} \int_{r_{H}}^{\infty} d r\left(4 \mu r r_{H}-2 \mu r^{2}\right)=0
$$

Thus, in this case in order to derive the correction to the black hole temperature resulting from the classical electric field outside the horizon, we need go to higher orders in $A, B$ and $m / M_{B H}$. 
Of course, in the case of unbroken electromagnetism one can derive an exact result. Plugging equation (11) into equations (5) and (6), we get

$$
\begin{array}{r}
\beta \hbar=4 \pi r_{H} \frac{1}{1+G \Phi} \\
r_{H}=2 G M_{B H} \frac{1}{1-G \Phi}
\end{array}
$$

with $\Phi$ defined by

$$
\Phi=\left(\frac{\omega}{\beta \hbar e}\right)^{2}
$$

In this case, $\Phi$ is the electric potential at the horizon, and the above equation then gives the relation between $\omega$ and the electric charge $Q$.

From this, it is straightforward to derive:

$$
M_{B H}=\frac{\beta \hbar}{8 \pi G}\left(1-G^{2} \Phi^{2}\right)
$$

and thus

$$
\begin{aligned}
\beta \hbar & \approx 8 \pi G M_{B H}\left(1+\left(\frac{\omega}{8 \pi e}\right)^{4} \frac{1}{G^{2} M_{B H}^{4}}+\cdots\right) \\
& \sim 8 \pi G M_{B H}\left(1+O\left(\frac{M_{p l}^{4}}{M_{B H}^{4}}\right)+\cdots\right)
\end{aligned}
$$

This is the Euclidean version of the well known result that classical electrically charged black holes have a lower temperature, for fixed mass, than their uncharged counterparts.

Note that the action can also be evaluated to lowest order in $G \Phi$, or equivalently $\left(M_{p l} / M_{B H}\right)^{2}$, (using the fact that $R=0$ outside the event horizon):

$$
\begin{aligned}
S & =\frac{(\beta \hbar)^{2}}{16 \pi G}\left[1+G\left(\frac{\omega}{\beta \hbar e}\right)^{2}\right]^{2} \\
& \sim \frac{(\beta \hbar)^{2}}{16 \pi G}+O(1)+O\left[\frac{M_{p l}^{2}}{M_{B H}^{2}}\right]+\cdots
\end{aligned}
$$

This result will also have some significance later in our analysis. 


\section{Discrete Charge and the Effect of Quantum Hair}

Now we consider the Euclidean Einstein-Abelian-Higgs system which, as described earlier, provides the prototypical example of quantum hair.

The action of this system is:

$$
S_{E}=\int d^{4} x \sqrt{g}\left(\mathcal{L}_{a h}-\frac{1}{16 \pi G} R\right)+\frac{1}{2}(\beta \hbar) M_{B H}
$$

with

$$
\mathcal{L}_{a h}=\frac{1}{4 \pi}\left[\frac{1}{4} g^{\mu \lambda} g^{\nu \sigma} F_{\mu \nu} F_{\lambda \sigma}+g^{\mu \nu}\left(D_{\mu} \phi\right)^{*}\left(D_{\nu} \phi\right)+\frac{\lambda}{4}\left(|\phi|^{2}-v^{2}\right)^{2}\right]
$$

The above action has solutions corresponding to a vortex sitting in the 2-d Euclidean $r-t$ plane of a black hole (i.e. see also [19]). The two other Euclidean dimensions $\theta, \phi$, (which would correspond to $z, t$ for a corresponding vortex in Minkowski space) are suppressed. As emphasized by CPW, in a Euclidean path integral formalism these instanton solutions play a central role in producing the observable effects of discrete charge outside of the black hole event horizon, as the sum over these instantons includes Aharonov-Bohm phases which are sensitive to the discrete charge contained the black hole. As we have described earlier, we can explore the effects on temperature of the individual instantons utilizing our formalism.

We use standard ansatz for these vortices:

$$
\begin{array}{r}
\phi=v f(r) e^{-i \frac{2 \pi}{\beta \hbar} t}, \\
A_{t}=\frac{2 \pi}{\beta \hbar} \frac{1}{e}(1-a(r)),
\end{array}
$$


with boundary conditions:

$$
\begin{aligned}
& f\left(r_{H}\right)=0, \quad f(\infty)=1 \\
& a\left(r_{H}\right)=1, \quad a(\infty)=0
\end{aligned}
$$

Now $A_{t}$ satifies an equation similar to that presented for the Reissner-Nordstrom solution. However, in this case, the quantization condition comes about simply as a reflection of the flux quantization condition for vortices in the broken phase:

$$
\left.e \int_{0}^{\beta \hbar} d t A_{t}\right|_{r=\infty}=2 \pi
$$

(in other words, $\omega=2 \pi$, rather than being a free parameter as it was in the Reissner-Nordstrom case).

We can also write down the action for the Higgs sector:

$$
\begin{aligned}
S_{\text {vortex }} & =4 \pi(\beta \hbar) \int_{r_{H}}^{\infty} r^{2} d r e^{-\phi(r)}\left[\frac{1}{2}\left(\frac{2 \pi}{\beta \hbar e}\right)^{2} a^{\prime 2}(r) e^{2 \phi(r)}\right. \\
& +\left(1-\frac{b(r)}{r}\right)^{-1} e^{2 \phi(r)} v^{2}\left(\frac{2 \pi}{\beta \hbar}\right)^{2} a^{2} f^{2} \\
& \left.+\left(1-\frac{b(r)}{r}\right) v^{2} f^{\prime 2}(r)+\frac{\lambda}{4} v^{4}\left(f^{2}-1\right)^{2}\right]
\end{aligned}
$$

Following CPW one can consider the two limiting cases of the above action, depending upon whether the vortex width is much larger or smaller than the size of the event horizon. Equations (9) and (10) lend themselves directly to such an analysis. Competition among the different terms as their $r$-dependence varies, can lead, in different limits, to a different sign for the correction to the black hole temperature. However, what actually occurs depends subtlely yet crucially on diffferences between the vortex solution in flat and curved space, as we shall describe in detail here. 


\subsection{Thin String Limit}

In the thin string limit, the vortex width $r_{s} \ll r_{H}$, so in (10),

$$
\begin{aligned}
2(A+B)-\frac{m}{M_{B H}} & \approx \frac{8 \pi G}{r_{H}} \int_{r_{H}}^{\infty}\left(4 \mu r r_{H}-2 \mu r^{2}\right) d r \\
& \approx 16 \pi G r_{H} \int_{r_{H}}^{\infty} \mu d r
\end{aligned}
$$

Thus the correction to $\beta \hbar$ due to the vortex instanton can be expressed as:

$$
\beta \hbar=8 \pi G M_{B H}\left[1-16 \pi G r_{H} \int_{r_{H}}^{\infty} \mu d r\right]
$$

In this limit, the vortex lies in the region $r \sim r_{H}$, where the Euclidean Schwartzchild metric

$$
d s^{2}=\left(1-\frac{r_{H}}{r}\right) d t^{2}+\left(1-\frac{r_{H}}{r}\right)^{-1} d r^{2}+r^{2} d \Omega^{2}
$$

can be well approximated by (note that our use of $\rho$ and $\tau$ in this specific example follows standard conventions, and these co-ordinate quantities are not to be confused with the quantities $\rho$ and $\tau$ used elsewhere throughout this article):

$$
d s^{2}=\rho^{2} d \tau^{2}+d \rho^{2}+r_{H}^{2} d \Omega^{2}
$$

with

$$
\rho^{2}=4 r_{H}\left(r-r_{H}\right), \quad \tau=\frac{2 \pi}{\beta \hbar} t
$$

In the space with coordinates $\rho$ and $\tau$, the vortex actually lives in flat space and

$$
r_{H} \int_{r_{H}}^{\infty} \mu(r) d r \stackrel{\rho_{2}=4 r_{H}\left(r-r_{H}\right)}{\longrightarrow} \frac{1}{2} \int_{0}^{\infty} \mu(\rho) \rho d \rho=\frac{1}{4 \pi} T_{\text {vortex }} \sim v^{2}
$$


where $\mu(\rho)$ is just the energy density of the Nielsen-Oleson vortex and $T_{\text {vortex }}$ is the tension of the vortex in flat space. It is then straightforward to see that:

$$
\begin{aligned}
A & \sim B \sim \frac{m}{M_{B H}} \sim \frac{1}{M_{B H}} \int_{r_{H}}^{\infty} \mu r^{2} d r \\
& \sim \frac{r_{H}^{2}}{M_{B H}} \int_{r_{H}}^{\infty} \mu d r \\
& \sim \frac{r_{H}}{M_{B H}} \int_{0}^{\infty} \mu(\rho) \rho d \rho \\
& \sim G v^{2} \sim \frac{v^{2}}{M_{p l}^{2}}
\end{aligned}
$$

which is consistent with the expansion leading to (9). An estimate of $r_{s}$ follows from

$$
r_{H} r_{s} \sim \rho_{s}^{2} \sim \frac{1}{v^{2}} \rightarrow r_{s} \sim \frac{1}{v^{2} r_{H}}
$$

where $\rho_{s}$ is the width of the vortex in the $\rho$ coordinate.

So the Hawking temperature can finally be expressed as:

$$
\begin{aligned}
\beta \hbar & =8 \pi G M_{B H}\left[1-16 \pi G r_{H} \int_{r_{H}}^{\infty} \mu d r\right] \\
& =8 \pi G M_{B H}\left[1-4 G T_{v o r t e x}\right] \\
& \sim 8 \pi G M_{B H}\left[1-O\left(\frac{v^{2}}{M_{p l}^{2}}\right)\right]
\end{aligned}
$$

The effect of a single instanton in this limit is thus to raise the black hole temperature. This is the first explicit example of the fact that a semiclassical contribution by itself can violate the Weak Energy Condition, described earlier. That this instanton contribution violates the WEC is perhaps not so surprising. In the thin string case, the symmetry breaking scale is much 
smaller than the event horizon size so that effectively for all distances outside the horizon the symmetry is completely broken, and no classical remnants of the underlying $U(1)$ symmetry should be visible on such scales. We shall later contrast this to the thick string case.

Of course we remind the reader that the limitation of our analysis is that one can only treat a single instanton (a solution of the coupled EinsteinMaxwell equations) contribution and not a sum over instantons (not such a solution). The latter sum is required in order to find the net thermal effect of discrete charge on the black hole, as CPW did, by using the path integral summation with instantons weighted by the appropriate action. If one does the summation a la CPW, the interference between instantons and anti-instantons produces a net effect on the black hole temperature which is opposite in sign to that for a single instanton

Nevertheless, the single instanton contribution to the temperature which we calculate using the energy momentum formalism directly is identical with that determined by CPW in the thin string case based on estimating the deficit angle and the instanton action. With this in mind, and for later comparison purposes, we also present our estimate of the action of the vortex solution in this limit.

$$
\begin{aligned}
S & =\int d^{4} x \sqrt{g}\left(\mathcal{L}_{E}-\frac{1}{16 \pi G} R\right)+\frac{1}{2}(\beta \hbar) M_{B H} \\
& \doteq \frac{1}{2} \int d^{4} x \sqrt{g}(\rho+\tau)+\frac{1}{2}(\beta \hbar) \frac{\beta \hbar}{8 \pi G}\left(1+16 \pi G r_{H} \int_{r_{H}}^{\infty} \mu d r\right) \\
& \doteq 2 \pi \beta \hbar r_{H}^{2} \int_{r_{H}}^{\infty}(\rho+\tau) d r+\frac{(\beta \hbar)^{2}}{16 \pi G}\left(1+16 \pi G r_{H} \int_{r_{H}}^{\infty} \mu d r\right)
\end{aligned}
$$




$$
\begin{aligned}
& \doteq \frac{(\beta \hbar)^{2}}{16 \pi G}+\frac{1}{2}(\beta \hbar)^{2} r_{H} \int_{r_{H}}^{\infty}(2 \mu+\rho+\tau) d r \\
& \sim \frac{(\beta \hbar)^{2}}{16 \pi G}\left(1+O\left(\frac{v^{2}}{M_{p l}^{2}}\right)\right)
\end{aligned}
$$

In above, we have used $R=-8 \pi T_{\mu}^{\mu}$. As $\mu>|\rho|,|\tau|$, and because $\mu \sim \mathcal{L}_{E}>$

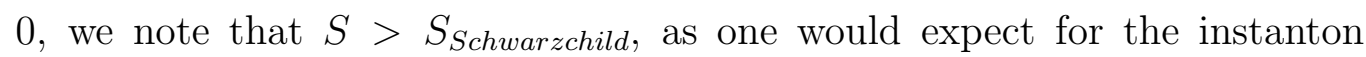
approximation to be stable.

\subsection{Thick String Limit}

The simplication which made the consideration of the thin string limit so straightforward was that one could simply picture the instanton as a vortex living in the two dimensions of a flat $r-t$ plane. The thick string limit, in which $r_{s}>>r_{H}$, is much more subtle. In this limit the curvature associated with the sphere at the event horizon cannot be ignored. If one were to ignore this curvature and just continue utilizing the flat space approximation one could then use well known properties of standard flat space vortex solutions inside the core, where the symmetry is unbroken, along with the boundary conditions associated with the magnetic flux carried in the core, to examine eq. (10), and estimate the instanton contribution.

An anomaly arises in this case, however, which signals that such a procedure is inconsistent. We have thus far presented the Euclidean action of each instanton solution. If we were to calculate the action of a flat space thick vortex, which we will present in more detail later, we would obtain a result which is nonsensical - namely that the instanton action is less than the Schwarzchild action. There is another worrisome result. The lowest order correction one would find to the black hole temperature in this case is 
proportional to $v^{2} / M_{p l}^{2}$, as it was in the thin string case, while CPW argue that a zeroth order contribution in this limit dominates.

Remarkably, as we now demonstrate explicitly, a proper consideration of the full curved space vortex solution in the thick string limit resolves the first problem, but the second problem persists even in this case.

The point is that if we consider the effects of curved space, associated with the spherical surface at the event horizon, the vortex core behaves very differently from the flat space vortex. Inside a flat space vortex, the symmetry is unbroken. In this case therefore one might imagine that the correct approximation is simply the unbroken theory, i.e. the Reissner-Nordstrom solution. However, the core of a long straight vortex in flat space looks quite different from vacuum electromagnetism in three dimensions. The only vacuum solution with the correct boundary condition, associated with the turn-on of the Higgs field at the vortex surface even if that surface is removed to infinity, involves a uniform magnetic flux in the core (i.e. the vacuum solution in two dimensions). In such a configuration the gauge potential and magnetic field both behave quite differently from that described in section 3. However, when the curvature associated with the spherical event horizon is taken into account, as it must be when the string core size is larger compared to the event horizon size (the thick string limit), the presence of extra $r^{2}$ contributions in the spherical derivatives around the event horizon allow a vortex solution in which both the gauge potential and magnetic field fall off exactly as in the unbroken $3 \mathrm{~d}$ theory inside the core, so that the physics inside becomes largely insensitive to the boundary conditions associated with the Higgs field behavior at the vortex surface. In other words, as $v \rightarrow 0$ the 
thick string limit smoothly approaches the Reissner-Nordstrom case-there are no singular effects due to boundary conditions at infinity in the curved space solution.

The previous argument can be made more explicit by examining the action and equations of motion of the vortex, in both the thin and thick string limits. Define the quantities

$$
y=\frac{r}{r_{H}}, \quad \epsilon^{2}=2 e^{2} r_{H}^{2} v^{2}, \quad \beta_{0}=\frac{\lambda}{2 e^{2}}
$$

The thick string limit is taken by letting $\epsilon^{2}$ tend to zero. Using these variables the action of the vortex becomes:

$$
S_{v}=\frac{1}{4 \pi} \frac{2 \pi^{2}}{e^{2}} \int_{1}^{\infty} y^{2} d y e^{-\phi(y)} \mu_{y}
$$

where

$$
\begin{aligned}
\mu_{y} & =\left(32 \pi e^{2} r_{H}^{4}\right) \mu \\
& =a^{\prime 2}(y) e^{2 \phi(y)}+\epsilon^{2}\left(1-\frac{b(y)}{y}\right)^{-1} e^{2 \phi(y)} a^{2}(y) f^{2}(y) \\
& +4 \epsilon^{2}\left(1-\frac{b(y)}{y}\right) f^{\prime 2}(y)+\beta_{0} \epsilon^{4}\left(f^{2}-1\right)^{2}
\end{aligned}
$$

In the above $\left.b\right|_{y=1}=1$. The other components of the energy momentum tensors can be similarly witten in terms of variable $y$,

$$
\begin{aligned}
\rho=T_{t}^{t}= & \frac{1}{4 \pi} \frac{1}{8 e^{2} r_{H}^{4}}\left[a^{\prime 2}(y) e^{2 \phi(y)}-\epsilon^{2}\left(1-\frac{b(y)}{y}\right)^{-1} e^{2 \phi(y)} a^{2}(y) f^{2}(y)\right. \\
& \left.+4 \epsilon^{2}\left(1-\frac{b(y)}{y}\right) f^{\prime 2}(y)+\beta_{0} \epsilon^{4}\left(f^{2}-1\right)^{2}\right]
\end{aligned}
$$

The action and the components of energy-momentum tensor above are quite similar in form to those in the flat space. However, there are two notable 
differences: the integral measure now becomes $y^{2} d y$ instead of $y d y$ and there is an additional dimensionless parameter $\epsilon$ which measures the relative size of the horizon and the vortex.

In the thin string limit, $\epsilon^{2} \rightarrow \infty$, from equation (13), we can see that $f$ tends to be 1 everywhere except possibly around the origin $y=1$. To find the leading piece, we can ignore the back-reaction of the vortex to the background geometry (since we assume $v \ll M_{p l} \ll M_{B H}$.) and set $y=1$ whenever there is an explicit dependence in the action integral. The simplified action is:

$$
\begin{aligned}
S_{v} & =\frac{1}{4 \pi} \frac{2 \pi^{2}}{e^{2}} \int_{1}^{\infty} d y\left[a^{\prime 2}(y)+\epsilon^{2} \frac{1}{y-1} a^{2}(y) f^{2}(y)\right. \\
& \left.+4 \epsilon^{2}(y-1) f^{\prime 2}(y)+\beta_{0} \epsilon^{4}\left(f^{2}-1\right)^{2}\right]
\end{aligned}
$$

The results of the previous section can be recovered when we change the integration variable in the action to $x$, defined as $x^{2}=4 \epsilon^{2}(y-1)$, in terms of which, the dependence of the action on $\epsilon$ factors out, and the action becomes:

$$
S_{v}=4 \pi r_{H}^{2} T_{N O}
$$

where $T_{N O}$ is the standard Nielsen-Oleson action for the vortex in two dimensional flat space. Note that in equation (14), the prefactor $r_{H}^{2}$ comes from the factored-out $\epsilon^{2}$ piece and the $v^{2}$ in $\epsilon^{2}$ has been absorbed into $T_{N O}$. Equation (14) is nothing but

$$
S_{v}=(\text { Worldsheet Area }) T_{\text {string }},
$$

as expected [5].

As $\epsilon^{2}$ becomes smaller and smaller, the vortex becomes thicker and thicker compared to the size of the event horizon, and from equation (13), it is 
clear that as the $\epsilon^{2}$ goes to zero, the action reduces to that of Euclidean Reissner-Nordstrom black hole. To see that the transition from the thick string limit to Reissner-Nordstrom case is smooth, i.e. there are no singular effects encountered due to boundary conditions from symmetry breaking at infinity, let us look at the equations of motion for the vortex. These are: (again here we ignore the back-reaction of the vortex)

$$
\begin{gathered}
a^{\prime \prime}+\frac{2}{y} a^{\prime}=\epsilon^{2}\left(1-\frac{1}{y}\right)^{-1} a f^{2} \\
\frac{1}{y^{2}}\left[y^{2}\left(1-\frac{1}{y}\right) f^{\prime}\right]^{\prime}=\frac{1}{4}\left(1-\frac{1}{y}\right)^{-1} a^{2} f+\frac{1}{2} \beta_{0} \epsilon^{2}\left(f^{2}-1\right) f
\end{gathered}
$$

The leading piece of $a(y)$ can be found by solving equation (15) with $\epsilon=0$, which is $a(y)=1 / y$, as expected [5]. Note the $y^{2}$ in the integral measure has made the gauge potential $a(y)$ behave like a three dimensional vacumm electromagnetic potential, resulting an electric field falling off as $1 / r^{2}$. It is instructive here to recall the equation of motion for the vortex in two dimensional flat space. The equation for the gauge potential is:

$$
a^{\prime \prime}-\frac{1}{r} a^{\prime}=f^{2} a
$$

As we take $f=0$, the equation above reduces to that for electromagnetism in two dimensional space, where $a \propto r^{2}$ and the magnetic field (in this case given by $\left.a^{\prime} / r\right)$ is constant over space. This is the familiar vortex core behavior in flat space. If it persisted in curved space, then the thick string limit would have a singularity associated with the surface at infinity which could not be ignored.

For a small but non-vanishing $\epsilon$, we can estimate the range in which $a(y) \sim 1 / y$ by looking at when the term proportioanl to $\epsilon^{2}$ in equation (15) 
becomes comparable to the other terms.

$$
a^{\prime \prime} \sim \epsilon^{2} \frac{y}{y-1} a f^{2} \Longrightarrow \frac{1}{y^{3}} \sim \epsilon^{2} \frac{y}{y-1} \frac{1}{y} \Longrightarrow y \sim \frac{1}{\epsilon}
$$

where we have used the fact that $a \sim 1 / y$ and $f \sim O(1)$. Thus the piece proportional to $\epsilon^{2}$ becomes important only after $y$ is of the same order as or larger than $1 / \epsilon$, so that the range in which $a$ behaves as $1 / y$ increases as $1 / \epsilon$ with $\epsilon$ going to zero, and the limit is indeed continous. Note that while the term of the order $\epsilon^{2}$ in equation (15) is proportional 1/(y-1), an analysis of equation (16) implies that around $y=1, f \propto \sqrt{y-1}$, so around $y=1$ the $f^{2}$ piece will cancel the divergence in $1 /(y-1)$ so there is no singularity.

It might seem from this argument that one should ignore the details of the $v$-dependent corrections to the black hole temperature in the thick string limit, as indeed was advocated by CPW. However we have argued in our previous work, and we demonstrate explicitly below, that this is not always consistent. Moreover, we will also demonstrate that while first order corrections in $\epsilon^{2}$ to the temperature can dominate, the first order contribution to the action is always negligible, and so the instanton action is always greater than the Schwarzchild action, as is required for consistency.

As the vortex becomes thicker and thicker with $\epsilon^{2}$ smaller and smaller, the mathemtical content of the above discussion is that in this limit there are no small or large parameters other than $\epsilon^{2}$ in the action and the energy momentum tensor. We can thus expand these quantities in terms of $\epsilon^{2}$ :

$$
\begin{aligned}
S_{\text {matter }} & =S_{0}+\epsilon^{2} S_{1}+\cdots \\
\rho & =\rho_{0}+\epsilon^{2} \rho_{1}+\cdots
\end{aligned}
$$




$$
\begin{aligned}
\tau & =\tau_{0}+\epsilon^{2} \tau_{1}+\cdots \\
\mu & =\mu_{0}+\epsilon^{2} \mu_{1}+\cdots
\end{aligned}
$$

The contributions from the zeroth order terms are identical to those in the case of the Euclidean Reissner-Nordstrom black hole, simply replacing $\omega$ by $2 \pi$, so that in this case the Hawking temperature and the action are given by,

$$
\begin{aligned}
\beta \hbar & \approx 8 \pi G M_{B H}\left[1+\left(\frac{1}{4 e}\right)^{4} \frac{1}{G^{2} M_{B H}^{4}}+\cdots\right] \\
& \sim 8 \pi G M_{B H}\left(1+O\left(\frac{M_{p l}^{4}}{M_{B H}^{4}}\right)+\cdots\right) \\
S & =\frac{(\beta \hbar)^{2}}{16 \pi G}\left[1+G\left(\frac{2 \pi}{\beta \hbar e}\right)^{2}\right]^{2} \\
& =\frac{(\beta \hbar)^{2}}{16 \pi G}+\frac{\pi}{2 e^{2}}+\frac{\pi G}{4 e^{2}}\left(\frac{2 \pi}{\beta \hbar e}\right)^{2} \\
& \sim \frac{(\beta \hbar)^{2}}{16 \pi G}+O(1)+O\left[\frac{M_{p l}^{2}}{M_{B H}^{2}}\right]+\cdots
\end{aligned}
$$

Here it is crucial that the lowest order correction to the Hawking temperature is of order $M_{p l}^{4} / M_{B H}^{4}$, instead of $M_{p l}^{2} / M_{B H}^{2}$ precisely because the lowest order contribution in $G \Phi$ to the temperature vanished in the unbroken theory (c.f. eq. (12)). Note that by comparison, the lowest order correction to the action is of $O(1)$.

The contributions from the first order terms (in $\epsilon^{2}$ ) can be found using eq (9) and (10).

$$
\begin{aligned}
2(A+B)-\frac{m}{M_{B H}} & =\frac{8 \pi G}{r_{H}} \epsilon^{2} \int_{r_{H}}^{\infty} d r\left[\left(4 \mu_{1} r r_{H}-2 \mu_{1} r^{2}\right)-\left(\rho_{1}-\tau_{1}\right) r\left(r-r_{H}\right)\right] \\
& =\frac{G}{4 e^{2} r_{H}^{2}} \epsilon^{2} T
\end{aligned}
$$


where

$$
T=\int_{1}^{\infty} d y\left[\left(4 \mu_{y 1} y-2 \mu_{y 1} y^{2}\right)-\left(\rho_{y 1}-\tau_{y 1}\right) y(y-1)\right]
$$

and $\mu_{y 1}, \rho_{y 1}, \tau_{y 1}$ are dimensionless quantities obtained from the corresponding unsubscripted quantities by extracting an overall dimensionful scaling factor $1 /\left(4 \pi\left[8 e^{2} r_{H}^{4}\right]\right)$.

In this case the expressions for the Hawking Temperature and the action are:

$$
\begin{aligned}
& \beta \hbar=8 \pi G M_{B H}\left(1-\frac{G}{4 e^{2} r_{H}^{2}} \epsilon^{2} T+\cdots\right) \\
S= & \int d^{4} x \sqrt{g}\left(\mathcal{L}_{E}-\frac{1}{16 \pi G} R\right)+\frac{1}{2}(\beta \hbar) M_{B H} \\
\approx & \frac{1}{2} \int d^{4} x \sqrt{g}(\rho+\tau)+\frac{1}{2}(\beta \hbar) \frac{\beta \hbar}{8 \pi G}\left(1+\frac{G}{4 e^{2} r_{H}^{2}} \epsilon^{2} T\right) \\
\approx & 2 \pi \beta \hbar \int_{r_{H}}^{\infty}(\rho+\tau) r^{2} d r+\frac{(\beta \hbar)^{2}}{16 \pi G}+\frac{\pi}{4 e^{2}} \epsilon^{2} T \\
\approx & \frac{(\beta \hbar)^{2}}{16 \pi G}+\frac{\pi}{2 e^{2}}+\frac{\pi}{4 e^{2}} \epsilon^{2} T^{\prime} \\
\sim & \frac{(\beta \hbar)^{2}}{16 \pi G}+\frac{\pi}{2 e^{2}}+O\left(\frac{v^{2} M_{B H}^{2}}{M_{p l}^{4}}\right)+\cdots
\end{aligned}
$$

where in the above $\pi / 2 e^{2}$ comes from $\rho_{0}$ and $\tau_{0}$ and

$$
\begin{aligned}
T^{\prime} & =\int_{1}^{\infty} y^{2} d y\left(\rho_{y 1}+\tau_{y 1}\right)+T \\
& =\int_{1}^{\infty} d y\left[2\left(\tau_{y 1}-\mu_{y 1}\right) y^{2}+\left(4 \mu_{y 1}+\rho_{y 1}-\tau_{y 1}\right) y\right] \\
& \sim 1
\end{aligned}
$$

Now we can compare in detail the results from the zeroth order and the first order terms to the Hawking Temperature [16], and to the action. The 
ratio of the first order in $\epsilon^{2}$ contribution to the zeroth order in $\epsilon^{2}$ contribution to the Hawking Temperature (and also the ratio of the subdominant $\epsilon^{2} \neq 0$ correction to the subdominant $\epsilon^{2}=0$ corrections to the action) is

$$
\gamma=\frac{\epsilon^{2} M_{B H}^{2}}{M_{p l}^{2}}=v^{2} M_{B H}^{4} / M_{p l}^{6}
$$

Now, recall that the thick string limit is

$$
\epsilon^{2}=v^{2} M_{B H}^{2} / M_{p l}^{4} \ll 1 .
$$

Recall that for the semiclassical analysis of black hole thermodynamics to be meaningful, $M_{p l}^{2} / M_{B H}^{2}$ has to be very small. For sufficiently massive black holes, it is certainly possible for both $\epsilon^{2} \ll 1$ and $\gamma \gg 1$ (i.e. if $M_{p l}^{2} / M_{B H}^{2} \ll$ $\epsilon^{2}$ ), in which case the contributions from the the first order terms in $\epsilon^{2}$ cannot be neglected. For example, if we keep $\epsilon^{2}$ fixed but let $\left(M_{B H} / M_{p l}\right)^{2} \rightarrow \infty$ (which requires also making $v^{2} \rightarrow 0$ ), then $\gamma \rightarrow \infty$, so that while both the zeroth and first order contributions in $\epsilon^{2}$ to the Hawking Temperature go to zero, the first order piece becomes arbitrarily large compared to the second. Stated another way, the limit in which only the Reissner-Nordstrom piece is considered, as was done by $\mathrm{CPW}$, is not the generic thick string limit, but is rather the limit $\gamma \ll 1$.

Note that in the same limit, the first order in $\epsilon^{2}$ piece to the action dominates over the first order in $M_{p l}^{2} / M_{B H}^{2}$, both of these terms are subdominant compared to the positive zeroth order piece $\pi /\left(2 e^{2}\right)$ associated with the Reissner-Nordstrom action. As a result, the instanton action in the thick string limit is (independent of the magnitude of $\epsilon^{2}$ ) always greater than the Schwarzchild action, as required for the instanton semiclassical approximation to be stable. 
It may seem somewhat surprising that for sufficiently large black holes the first order terms in $\epsilon^{2}$ may become comparable or larger than the zeroth order terms. However, this is perhaps understandable when one considers the dependence of the detailed vortex solution on the curvature at the event horizon. Recall that for a flat space vortex, the field behavior inside the vortex core differs dramatically compared to that outside the event horizon of the Reissner-Nordstrom solution. It is only the curvature at the spherical event horizon which allows the vortex instanton to approach the ReissnerNordstrom solution in the thick string limit. Now, for larger black holes the curvature at the event horizon becomes progressively smaller. While it may be true that the $v \rightarrow 0$ limit always goes smoothly to the ReissnerNordstrom case, independent of the black hole mass, increasing the black hole mass reduces the curvature effects at the horizon which are responsible for the domination of the Reissner-Nordstrom contribution compared to the symmetry breaking contribution proportional to the vev of the Higgs field. Thus, one might expect that the value of $\epsilon^{2}$ must be correspondingly reduced as the black hole mass increases in order for the first order contribution in $\epsilon^{2}$ to be negligible. Put another way, the string must be correspondingly thicker (in relation to the size of the event horizon) as the black hole mass increases in order for the dominant contribution to the Hawking temperature of the instanton to be that approximated by the Reissner-Nordstrom solution.

Lastly we consider the possible sign of the first order term in the expression for the black hole temperature given above. It is clear from the expression for $T$ that this is in general indeterminate. However, if we make the ansatz that the first order term takes a form similar to that which would 
occur for the flat space vortex, which does not seem unreasonable, then, assuming the first order quantities all have support over a scale $r_{s} \gg r_{H}$ $(y \gg 1)$

$$
\begin{aligned}
T \rightarrow-\int_{1}^{r_{s}} & \left(2 \mu_{y 1}+\left(\rho_{y 1}-\tau_{y 1}\right)\right) y^{2} d y \\
& \rightarrow-2 / 3\left(r_{s} / r_{H}\right)^{3}<\mu_{y 1}>
\end{aligned}
$$

This is manifestly negative, and hence the contribution of this piece to the Hawking temperature would be of the same sign as the zeroth order contribution. To the extent that our anzatz is valid then, instantons in the thick string limit would generically cool down a black hole - the opposite of the thin string result. This result (obtained by CPW for purely the zeroth order piece) is again heuristically understandable. In the thick string limit, because the instanton approaches the Reissner-Nordstrom solution, it is to be expected that the effect on temperature will be that appropriate to the Reissner-Nordstrom solution - namely to cool down the black hole. Of course, again because the actual dependence on discrete charge comes from a sum over instantons and anti-instantons, the effect of discrete charge in this limit is to raise the black hole temperature. This result, which was a priori somewhat surprising, given the similarity of the thick string limit and the Reissner-Nordstrom case, is then understandable as being due to the similarity of the instanton and the Reissner-Nordstrom solution, combined with the fact that the sum over instantons in the case of discrete charge produces results in the opposite temperature correction compared to the single instanton contribution. 


\section{$5 \quad$ Non-Classical Electric Fields Redux}

Our analysis thus far has enhanced our understanding of the physical basis of the effects of instantons on black hole thermodynamics by concentrating on the energy-momentum tensor outside the event horizon associated with the instantons themselves. Of course, as we have indicated already, the limitation of this is first that it is not a single instanton but rather the sum over instantons which is finally relevant to the calculation of physically measurable effects, and second, that instantons only give relevant corrections to quantities which otherwise have no perturbative contributions, and which thus vanish in the classical limit.

Nevertheless, while focussing on instantons, it is worth recalling that the instanton solutions described here are not present in the Abelian-Higgs theory in flat space. Indeed, it is well known that there is no instanton in the Abelian-Higgs model in four dimensional flat spacetime since the Euclidean sector has the topology of $R^{4}$ or $R^{3} \times S^{1}$, which does not admit any finite energy solutions. In a $(1+1)$ dimensional spacetime, however, there are instantons which correspond to localized vortices in the $r-\tau$ plane. Now, as we have discussed, the black hole sector of the 4-d Einstein-Abelian-Higgs model has the topology of $R^{2} \times S^{2}$. Thus, the system admits a finite action instanton solution which corresponds to a vortex sitting in the $2-\mathrm{d} r-t$ plane $\left(R^{2}\right)$ of a black hole with the other two dimentions $\theta, \phi\left(S^{2}\right)$ suppressed. (It can be imagined as a Eulidean string with its worldsheet a sphere $S^{2}$. Because the string worldsheet $S^{2}$ is compact, the solution has finite action.) We have seen that the existence of these new instantons can affect the properties of the black hole. Moreover, besides the effect on black hole thermodynamics, 
there is an even more striking effect: the instanton sum results in a quantum mechanical electric field outside the event horizon of the black hole endowed with discrete hair [5, 6].

In this section we wish to comment on this aspect of the Euclidean instanton solutions described here, and their relation to instantons in the two dimensional Abelian Higgs model.

As emphasized by CPW, the partition function for a black hole in a charge $Q$ sector is given by 5 :

$$
Z(\beta, Q)=\operatorname{Tr}\left(P_{Q} e^{-\beta H}\right)=\int_{-\infty}^{\infty} d \omega e^{-i 2 \pi w Q / \hbar e} Z(\beta, \omega)
$$

where $P_{Q}$ is the charge projection operator and

$$
Z(\beta, \omega)=\int_{\beta \hbar, \omega} d A d \phi \exp \left(\frac{-S_{E}}{\hbar}\right)
$$

is the Euclidean path integral over configurations which are periodic in $\tau$ and satisfy the gauge constraint described earlier:

$$
\left.\frac{e}{2 \pi} \int_{0}^{\beta \hbar} d t A_{t}\right|_{r=\infty}=\omega
$$

(Recall that in the Higgs phase $\omega$ is quantized, with only integer values allowed. Thus the integral in eq (17) reduces to a summation over all integers. In this case, we can see from the phase factor in (17) that only the fractional part of $Q / \hbar$ is is physically meaningful.)

Restricting to the Euclidean $r-t$ plane, we can also write eq (18) as,

$$
\begin{gathered}
\omega=\frac{e}{2 \pi} \oint_{r-t} \vec{A} \cdot \overrightarrow{d l}=\frac{e}{4 \pi} \int_{r-t} d^{2} x \epsilon_{\mu \nu} F_{\mu \nu} \\
\mu, \nu=0,1
\end{gathered}
$$


where in the above $\vec{A}$ denotes the $t, r$ components $\left(A_{0}, A_{1}\right)$ of the vector potential $A$. Plugging (19) into (17), we get,

$$
\begin{gathered}
Z(\beta, Q)=\int_{\beta \hbar} d A d \phi \exp \left(\frac{-S_{E}}{\hbar}-i \frac{e \theta}{4 \pi} \int_{r-t} d^{2} x \epsilon_{\mu \nu} F_{\mu \nu}\right) \\
\mu, \nu=0,1
\end{gathered}
$$

We have defined $\theta$ as

$$
\theta=\frac{2 \pi Q}{\hbar e}
$$

and the integral is over all configurations periodic in $\tau$. (From the comment below eq (18), it is clear that in the broken phase, the theory is periodic in $\theta$ with period $2 \pi$.) Eq (20) shows that to get the partition function for a black hole in a charge $Q$ sector, instead of using the charge projection and $Z(\beta, w)$ in (17), we can simply integrate over all configurations, provided we use a modified $\theta$ dependent action given by (up to gauge fixing),

$$
S_{\theta}=\frac{S_{E}}{\hbar}+i \frac{e \theta}{4 \pi} \int_{r-t} d^{2} x \epsilon_{\mu \nu} F_{\mu \nu}, \quad \mu, \nu=0,1
$$

The additional term, is nothing but the familiar topological term in two dimensions with $\theta$ as the topological charge. Since the system is spherically symmetric, we expect the integration over spherically symmetric configurations would dominate the integral (20), in which case, the system then reduces completely to the corresponding model in $(1+1)$ dimensions with a topological $\theta$ term.

It is interesting to see here that the electric charge $Q$ of a black hole reduces, in leading approximation, to a topological charge in $(1+1)$ dimensions. While this correspondence seems surprising at first sight, it can be 
understood as follows. The Coulomb phase of a (4d) $U(1)$ theory respects a superselection rule labeled by electric charge $Q$ associated with a long-range electric field. ( $Q$ can be discrete (integer multiple of some basic unit $e_{0} \hbar$ ) or continous depending on whether the $U(1)$ symmetry is compact or not.) In the Higgs phase, although classically the superselection rule no longer holds due to the screening of the long-range electric field, quantum mechanically there is still a nontrivial superselection rule left, provided that the original $U(1)$ is non-compact, or, in the compact case, the condensation charge $e=N e_{0}$ with $N$ an integer other than 1 . The superselection sector now is labeled by $Q(\bmod \hbar e)$, associated with a nontrivial Aharonov-Bohm phase

$$
\exp \left(\frac{2 \pi i Q}{\hbar e}\right)
$$

at infinity. In terms of $\theta$ defined in (21), in the noncompact case, the system is labeled by a continuous parameter

$$
\theta=\frac{2 \pi Q}{\hbar e} \in(0,2 \pi)
$$

while in the compact case, it is a $Z_{N}$ charge,

$$
\theta \in Z_{N}=\{\exp (2 \pi k / N), k=0,1, \cdots N-1\}
$$

Now the two-dimensional system (with an unbroken or broken $U(1)$ ) also respects a superselection rule where superselection sectors are labeled by the topological charge $\theta$. The different superselection sectors fall into different $\theta$-vaccua which are accompanied by a constant background electric field (classical in the unbroken $U(1)$ theory, nonclassical in the broken $U(1))$. Now the correspondence between the electric charge $Q$ and the topological charge is clear: from a 4-d point of view, the superselection sector the black hole falls 
into is labeled by its electric charge $Q$. On the other hand, since the system is spherically symmetric, the theory reduces to a effective $2 \mathrm{~d}$ theory, where one can label the superselection sector by the topological charge associated with the topological term (22).

The correspondence between the black hole system here and 2-d models can also help us gain some insights into the existence of a quantum mechanical electric field outside the event horizon of a black hole with quantum hair first discovered by CPW [5, 6]. It just corresponds to the familiar fact that there is a nonclassical background electric field in the $(1+1)$ dimensional abelianhiggs model associated with instantons and the topological term. There is a notable difference, however. In the $(1+1)$ dimensional Abelian Higgs model, there is a dilute instanton gas - the instantons can sit at any point in the two dimensional Euclidean space - resulting in a constant electric field as the system (due to translational invariance). In the quantum hair case, however, since the existence of the instanton solutions depends crucially on the topology of a black hole, they can only sit at the event horizon of a black hole. Thus, instead of a dilute instanton gas, we only have a single localized instanton, resulting a localized electric field which dies off at large distance from the event horizon.

This exact analogy between discrete quantum numbers on black holes in four dimensions and topological quantum numbers on related two dimensional systems may be of some interest for those wishing to interpret black hole entropy in terms of underlying quantum numbers associated with state counting in theories in which the black hole is the low energy limit of a string theory. 


\section{Conclusions}

Our analysis, based on considerations of the energy momentum tensor of fields outside the event horizon has allowed us to calculate instanton corrections to the temperature of black holes. Utilizing this formalism, the competition between different terms in the energy momentum tensor is explicitly demonstrated to lead to either a heating up, or a cooling down of the black hole in a way which is physically transparent. In addition, we see how the Euclidean energy momentum tensor associated with the instanton fields outside the event horizon yields semiclassical corrections which can violate the weak energy condition. In the thin string limit our results are identical to those obtained by CPW. However, our analysis has allowed us to extend their results. In the thick string limit, the effects of symmetry breaking need not be sub-dominant, allowing us to reconcile our flat space intuition with the curved space results. This new correction probably does not result in a qualitative change in the picture, since our intuition also suggests that the first order contributions will have the same sign as the zeroth order contributions, but this remains to be quantitatively checked.

We stress again that the corrections which we calculate are themselves only relevant to quantities which vanish in the classical limit. In the case of discrete quantum hair, the instanton contributions are relevant because (a) there is no perturbative or classical signature associated with the quantum hair, and (b) the quantum phases associated with their contributions to Euclidean path integrals allow a non-zero result when a sum over instantons and anti-instantons is performed, as demonstrated by CPW. Nevertheless, our results indicate that one may fruitfully extend Minkowski space methods 
designed to probe the effects of classical fields outside the event horizon on the thermodynamical properties of black holes to the Euclidean regime of semiclassical phenomena. This allows a more intuitive physical picture of the origin of such effects. It may also be useful in exploring the nature of other semiclassical contributions to black hole thermodynamics beyond those considered here associated with quantum hair.

It would be very nice to be able to extend our analysis so that the Euclidean field outside the event horizon associated with the instanton sum could be treated directly, so that perhaps the relation between the nonclassical electric field outside the event horizon and the change in the black hole temperature could be directly linked.

Finally, returning to the traditional Euclidean partition function in the instanton approximation, we have been able to derive an exact analogy between discrete charge on black holes in four dimensions and topological charge in related two dimensional systems. Using this analogy, the non-classical electric field outside black holes endowed with discrete hair can be understood as merely a special case of the well known existence of a non-classical electric field in the spontaneously broken two dimensional Abelian Higgs model endowed with a topological term. This analogy may be useful in considerations of the relation between black hole entropy and state counting.

LMK would like to thank Frank Wilczek for conversations early on in this analysis, and Sidney Coleman and John Preskill for several long and very illuminating conversations which helped steer us away from several red herrings. Finally, LMK and HL thank Tanmay Vachaspati for discussions of strings in curved space and key insights which helped us resolve the details 
of the thick string limit. LMK and HL are supported by the DOE and funds from Case Western Reserve University.

\section{References}

[1] S. W. Hawking, Phys. Rev. D14, 2460 (1976)

[2] J. Preskill, Phys. Scrip. T36, 258 (1991)

[3] M. J. Bowick, S. B. Giddings, J. A. Harvey, G. T. Horowitz, and A. Strominger, Phys. Rev. Lett. 61, 2823 (1988)

[4] L. M. Krauss and F. Wilczek, Phys. Rev. Lett. 62, 1221 (1989)

[5] S. Coleman, J. Preskill, and F. Wilczek, Nucl. Phys. B387, 175 (1992)

[6] S. Coleman, J. Preskill, and F. Wilczek, Mod. Phys. Lett. A6, 1631 (1991), Phys. Rev. Lett. 67, 1975 (1991)

[7] J. D. Bekenstein, Phys. Rev. D5, 1239, 2403 (1972)

[8] C. Teitelboim, Phys. Rev. D5, 2941 (1972)

[9] S. L. Adler and R. B. Pearson, Phys. Rev. D18, 2789 (1978)

[10] A. E. Mayo, J. D. Bekenstein, gr-qc/9602057

[11] Y. Aharonov and D. Bohm, Phys. Rev. 119, 485 (1959)

[12] M. G. Alford and F. Wilczek, Phys. Rev. Lett. 62, 1071 (1989)

[13] J. Preskill and L.M. Krauss, Nucl. Phys. B341, 50 (1990) 
[14] M.G. Alford, J. March-Russell and F. Wilczek, Nucl. Phys. B337, 695 (1990)

[15] S. Coleman, L. M. Krauss, J. Preskill, and F. Wilczek, J. Gen. Rel. Grav., (1991)

[16] L. M. Krauss, H. Liu, and J. Heo, hep-th/9610135

[17] M. Visser, Phys. Rev. D46, 2445 (1992)

[18] J. M. Bardeen, B. Carter, and S. W. Hawking, Commun. Math. Phys. 31, 161 (1973).

[19] F. Dowker, R. Gregory and J. Traschen, Phys. Rev. D45, 2762 (1992) 